\title{
Discovering and transforming precipitate phases in aluminium alloys using in situ transmission electron microscopy
}

\author{
L. Bourgeois ${ }^{1,2}$, Z. Zhang ${ }^{3,4}$, Y. Zhang ${ }^{2}$, X. Tan ${ }^{2}$, Y. Chen ${ }^{5}$, M. Weyland ${ }^{1,2}$, P.N.H. Nakashima ${ }^{2}$, N.V. Medhekar ${ }^{2}$ \\ ${ }^{1}$ Monash Centre for Electron Microscopy, Monash University, Australia, ${ }^{2}$ Department of Materials Science and Engineering, Monash \\ University, Australia, ${ }^{3}$ Electron Microscopy for Materials Research, University of Antwerp, Belgium, ${ }^{4}$ Department of Materials, \\ University of Oxford, United Kingdom, ${ }^{5}$ Thermofisher Scientific, The Netherlands
}

laure.bourgeois@monash.edu

Many phase transformations associated with solid-state precipitation look structurally simple, yet take place with great difficulty. Classic cases of surprisingly difficult phase transformations can be found in alloy systems forming the basis for a broad range of high-strength lightweight aluminium alloys. In these systems, the difficult nucleation of strengthening phases, which are usually semi-coherent, is often preceded by the easy nucleation of another phase with strong structural similarities, typically a coherent precipitate. It is therefore of interest to investigate the reasons behind the difficult transformation from coherent to semi-coherent precipitate phases.

Using scanning / transmission electron microscopy (S/TEM) techniques both ex situ and in situ, combined with atomic scale simulations (density functional theory and semi-empirical potentials) we examined phase transformations in several alloy systems, including the textbook $\mathrm{Al}-\mathrm{Cu}$ and $\mathrm{Al}-\mathrm{Ag}$ systems. We show that certain microalloying additions, or different processing conditions applied to samples in bulk or nanoscale form, result in previously unreported precipitate phases [1-2] - see Figs. 1-2, or promote the nucleation of existing phases [3-4]. The nucleation mechanisms of these phases involve structural templates provided by coherent precipitates [1-3] and depend critically on the availability of vacancies [1-2,4]. Based on our observations atomic-scale mechanisms are proposed for phase transformation pathways. We also characterised the surface structure and growth mechanisms of voids, uncovering a crystallographic relationship necessary for the growth of high-aspect ratio voids [5]. These findings suggest several approaches to not only stimulate known precipitate transformations, but also discover new phases and transformation pathways.

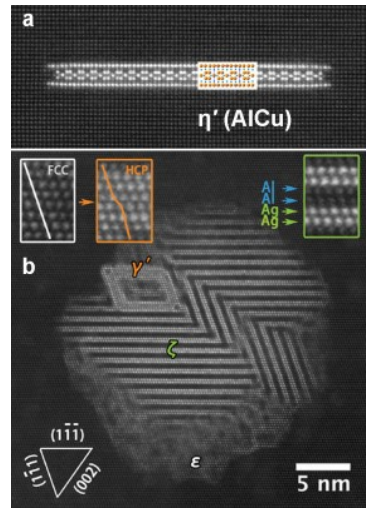

Figure 1. New phases discovered in the (a) Al-Cu and (b) Al-Ag alloy systems via in situ TEM: (a) the $\eta^{\prime}$ phase, $\mathrm{AlCu}[2]$, and (b) the $\zeta$ phase, $\mathrm{AlAg}[1]$.

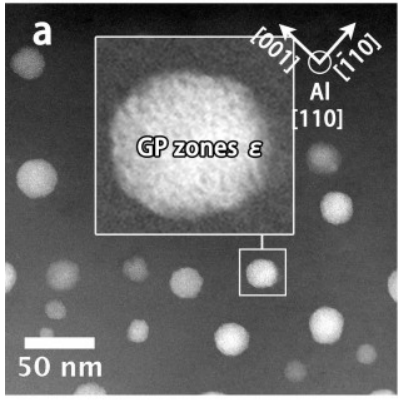

Before in situ

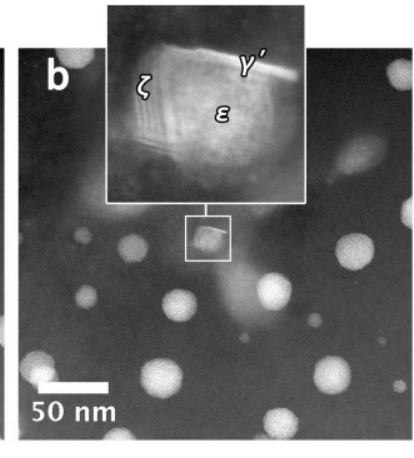

After in situ
Figure 2. In situ heating in the TEM showing the transformation of a Ag-rich particle (GP zone) into the $\zeta$ phase and the known $\gamma^{\prime}$ phase [1].

[1] Zhang, Z., Bourgeois, L., Rosalie, J. M. \& Medhekar, N. V. (2017). Acta Mater. 132, 525.

[2] Bourgeois, L., Zhang, Y., Zhang, Z., Chen, Y. \& Medhekar, N. V. (2020). Nature Comm. 11, 1248.

[3] Chen, Y. et al. (2017). Acta Mater. 125, 340.

[4] Zhang, Y., Zhang, Z., Medhekar, N. V. \& Bourgeois, L. (2017). Acta Mater. 141, 341.

[5] Tan, X., Weyland, M., Chen, Y., Williams, T., Nakashima, P. N. H. \& Bourgeois, L., (2021). Acta Mater. $206,116594$.

\section{Keywords: crystal discovery; solid-state precipitates; in situ transmission electron microscopy; atomistic simulations}

We thank Dr Julian Rosalie and Prof. Jiehua Li for providing alloy samples. We also acknowledge the Australian Research Council, computational support MonARCH, MASSIVE, and the National Computing Infrastructure and Pawsey Supercomputing Centre, and the Monash Centre for Electron Microscopy, a node of Microscopy Australia. 